\title{
Evolutionary Based on Selfish and Altruism Strategies - An Approach to Path Planning Problems
}

\author{
Paulo Salgado \\ CITAB/ECT Depart. de Engenharias \\ Universidade de Trás-os-Montes e Alto \\ Douro \\ Vila Real, Portugal \\ psal@utad.pt
}

\author{
Getúlio Igrejas \\ Escola Superior de Tecnologia e \\ Gestão Instituto Politécnico de \\ Bragança, \\ Bragança, Portugal \\ igrejas@ipb.pt
}

\author{
Paulo Afonso \\ Instituto de Telecomunicações \\ ESTGA, Universidade de Aveiro \\ Agueda, Portugal \\ pafnaa@ua.pt
}

\begin{abstract}
This paper presents a novel hybrid optimization approach based on a genetic algorithm that combines selfish gene and altruism view of evolution. The purpose of the present research is to develop a new optimization approach to solve path-planning problems, particularly to be used in robot trajectories planning. A brief discussion about selfish versus altruism is made in the perspective of genes, its integration in the chromosome (individuals) and the forces involved in the evolution process of genes, individuals and populations. The SAGA (Selfish-Altruist Genetic Algorithm) is the generalization of the Genetic Algorithms (GA), where the basic variables are the genes (characters or words) as nonautonomous entities, grouped in a Chromosome structure. The proposed hybrid approach was applied to a path-planning problem, in a continuous search space, to show its effectiveness in complex and interdependent sub-paths and evolution processes. Genes-centred evolution improved local sub-paths as sub-processes in a Chromosome-centred evolution and resulted in improved global planning trajectories when compared with standard genetic algorithm.
\end{abstract}

Keywords-Evolutionary algorithm, selfish, altruism, genetic algorithm.

\section{INTRODUCTION}

The theory of evolution is a new light in human thought and it has today transposition to computational models. Successive Evolutionary Algorithms (EA) and artificial life have been presented during the last 4 decades through computational simulations to solve complex engineering problems [1], particularly in optimization. Genetic algorithms have become one of the most famous EA algorithms since the pioneering work of John Henry Holland at 1975 [2]. They are based on Darwin's natural selection theory and were extended today to the automatic evolution of computer programs [1]. These evolutionary algorithms are now used to solve complex multi-dimensional problems more efficiently than other traditional methods, which do not own appropriate features that restrict their general use [3][4].

Evolution by natural selection is a process not only demonstrated by the observation of fossils, but also by phenotypic variation of living beings. These traits confer different performances in a severe and competitive environment. By the reproductive mechanism, these characteristics can be transferred from generation to generation [5] making the traits that favor their Fitness Function (FF) more common in the next generation. This results from the fact that offspring which parents have good FF (more survival capacity and high reproductive rate) will, most probably, acquire their parent's traits that will give them a competitive advantage to survive and reproduce. So, for natural selection is necessary and sufficient to have three conditions [6]:

- Diversity: Individuals within a population have different characteristics/traits (or phenotypes).

- Inheritance, a combination of parent's traits is transferred to offspring.

- Competition and reproduction: Individuals possessing traits well suited for the struggle for local resources will contribute more to the next generation offspring.

However, due to a high number of offspring and limited local resources, there is a substantial mortality. An overview of the basic process of natural selection can be found in [5] [7].

Since 1953 it is known that genes are encoded in a DNA molecular chain and that they control hereditary traits. Various processes or evolutionary mechanisms promote genetic changes from one generation to another [8]. The four most widely recognized of them are natural selection, mutation, genetic drift and gene migration due to genetic addition [9]. The first two result in new genetic ordering while gene mutation and migration create genetic variation. Hereditary traits are transferred from one generation to the next via DNA very faithfully over many generations. The DNA sequences can change through mutations, producing new alleles and affecting the characteristics of the underlying gene, altering the phenotype. It is known now that there are genetic changes that cannot be explained by order changes in the nucleotide sequence of the DNA.

In the evolutionary process described above, it is assumed that the unique participatory entity in the evolutionary process is the individual (an integral unit of a population where it has its own identity), characterized by its unique chromosomes. The individual is the only element that is selected to participate in the reproductive process, only favored by the set of its own characteristics revealed in the performance tests.

In another point of view about the evolution process, Richard Dawkins in his book "The Selfish Gene" (1976) defines the gene as a unit of selection [10][11]. Additionally, in his book "River Out of Eden", he says that in nature only genes have an utility function to replicate themselves in order to secure and perpetuate their own existence. For him, individual organisms contribute weakly to the evolution process, behaving as mere carriers of genes, their factory and their host, with the function of testing their merits or faults. Selected milestones in the study of selfish genetic elements are presented in [10] (pages, 12-13) and [12]. For all of them, 
genes are the basic entity replicators with the following properties:

- Longevity - it survives long enough to replicate or make copies of itself.

- Fecundity - at least one version of it can replicate.

- Fidelity - even after several generations of replications, it is still almost identical to the original.

This work adopts the principle that both evolutionary processes occur simultaneously, being a single process with two interacting mechanisms [13][14]. The evolutionary process uses the individual according to the principles of natural evolution by measuring it by its phenotype characteristics. In this case, the genes act together, admittedly in a context of altruistic behavior between genes in favor of individuals. At the same time, it operates genes in an effort to replicate their genetic code.

However, this is a virtual separation. Individuals are not the owners of the chromosomes but rather entities that carry genes borrowed by the species. On the other hand, genes do not have reproductive autonomy and they do not work alone.

The evolutionary mechanism uses two concurrent processes. In one, the adaptations mechanism promotes the phenotypic effects of genes in order to maximize their representation in the future generations. This is made by increasing the survival probability of those alleles remain in genes population whose phenotypic effects benefit the individuals, by successfully promoting their own propagation. In parallel, the individuals promote its best genes and are selected for the reproduction pool according to its fitness. Genes are the elementary elements that belong to the population of chromosomes and to the population of individuals, which share the genetic code inheritance of the species. The genetic code of the species is the combination of genes and its chromosomic structure.

In such processes, it is not required that individuals and genes belong to separate entities and subjected to a different performance test evaluation. An individual affirms their identity by the sum of their genes traits and only during their lifetime existence, stressing the performance of their genes (not always testing all of them) under casual environmental conditions to which it was subjected.

In contrast, genes are agents involved in the reproductive process, subject to a set of genetic operations, and participating in this process with their own identity and a relevant weight, given by their accumulated performance from several past generations (even if not revealed in the life of the individual). In the first situation, the good/poor performance of the individual favors/disturbs its probability to survive and reproduce, transposing/eliminating his genetic traits for the next generation. In the second case, it is evaluated the best contribution that an individual set of genes can offer for the future of the species, perpetuating it or not in the genes pool [15].

In this paper, this new evolutionary paradigm was tested in the problem of trajectories planning (in continuous space). It is intended to find a sequence of line segments that establish a connection between the point of departure and the point of arrival, without overlapping obstacles scattered in the navigation region [16].

This problem promptly reveals the difficulties of the conventional GA to do a feasible solution, namely due to its (premature) convergence, often being stuck in an insanitary solution within the search space. It is still an N-complex problem type. The SAGA algorithm, by combining an individuals and genes evolutionary strategy, offers a best solution for this kind of problems and eliminates the GA weakness.

This paper is organized as follows. Section 2 presents the main structure of the Selfish-altruist Genetic Algorithms (SAGA). The path-planning problem is described in Section 3. The main results of SAGA algorithm applied to solve the path-planning problem is presented in section 4 . They are a first group of results involving these new evolutionary strategies. Its performance is also described in this section. The SAGA algorithm results are compared with GA results. Finally, the last section presents the main conclusions of this study and proposed algorithm.

\section{Selfish-Altruist Genetic Algorithm}

As the GA, the Selfish-Altruist Genetic Algorithms (SAGA) use one group of individuals that are possible solutions for the optimization problem. These individuals are codified by own chromosomes, which contains genes. Their main objective is iteratively to modify them in order to obtain a renewed population with best genes, for best chromosomes that made best individuals. So, both components are evaluated in their fitness at each generation. For the gene the best value of the fitness of the individuals who are carrying it in the present or previous generations is given by:

$$
F G_{g}^{(k)}=\max \left(F G_{g}^{(k-1)}, \max _{i \supset g} F I_{i}^{(k)}\right)
$$

where $F G_{g}^{(k)}$ is the fitness value of gene $g$ in $k^{\text {th }}$ generation and $F I_{i}^{(k)}$ is the fitness of the $i^{\text {th }}$ individual that contains the gene $g$ in its chromosome, i.e. $i \supset g$.

The genes fitness must be adjusted in the following cases:

- If $g$ mutates then $F G_{g}^{(k)}=\max _{i \supset g} F I_{i}^{(k)}$

- Let $S_{g h} \in[0,1]$ a similarity factor between gene $g$ and gene $h$. If $S_{g h}>S_{\text {min }}$ and $F G_{h}>F G_{g}$, then $F G_{g}^{(k)}=F G_{g}^{(k)}\left(1-S_{g h}\right)+S_{g h} F G_{h}^{(k)}$, with $S_{\text {thresh }}$ the thresholder of similarity.

The individuals of populations are evaluated in two modes. First, through the fitness function (by an altruism mechanism of chromosomes). Second, by an average combination of the fitness values of one part of their best genes (the selfish mechanism of genes).

The processes of the proposed SAGA are as follows:

Step 1. Create the initial population of $\mathrm{n}$ individuals.

Step 2. Evaluate the population through the fitness function. Calculate the fitness of genes contained in the populations with eq. (1).

Step 3. Crossover parent's chromosomes to produce a child solution. Additionally, best genes have more probability to be transferred to the offspring than genes with poor fitness. Genes transferred carry its performance value. 
Step 4. Apply mutation operator on each chromosome of current population (by using a set of mutation operators). With WeigthedGenes Mutation function, genes with poor fitness have more probability to mutate. Evaluate the mutated result through the fitness function. Update the fitness of genes.

Step 5. Choose $n$ (population size) chromosomes of the best quality (individual with good performance or individual with a large set of good genes) from the newly generated chromosomes. From this pool, $\mathrm{n}$ selected chromosomes create the next generation of individuals.

Step 6. Repeat step 2-step 5 until the specified number of generations or the maximum iteration number is reached.

Tournament Selection and Roulette Selection are used processes of selecting parents to the reproduction mechanism and create offspring for the next generation. Each one is responsible for half of the selected individuals. The Natural and Real crossover are used for mate and recombine to create new chromosomes. Four mutation operators are used: Uniform, Border, Perturbation and WeigthedGenes, with the last used only by SAGA. All of these methods and operators are well described in literature and are part of most practical implementations of the evolutionary algorithms [17].

Both GA and SAGA are used to solve a same pathplanning problem. Each gene consists of a sequence of pairwise positive real values that represent a node of the trajectory. A chromosome is a sequence of genes, i.e. a sequence of waypoints of the path. Structures with one or multiple population (with migration facilities) are tested for this problem.

\section{The Path-Planning Problem}

Here, the path-planning problem is formulated as the task of determining a continuous path that connects two specified locations, a start and an end-point, while avoiding collision with known obstacles, if possible doing this within a safety clearance distance. Without prejudice to the use of other forms of trajectory, in this work, the path joining the starting point and the point of arrival will be constructed from the connection between $\mathrm{n}$ successive and ordered waypoints (nodes) located in the working region, joined by a chain of straight-line segments in a 2D space. $T=\left\{P_{0}, P_{1}, \cdots, P_{n}, P_{\text {end }}\right\}$ is the ordered set of the nodes $\mathrm{P}$ of the trajectory. A segment $S_{i}=\overline{P_{i-1} P_{i}}$ also straight segments, where $O$ is the set of their pairs of endpoints.

The main objective of path-planning algorithms is to find the optimal ordered nodes $\mathrm{T}$ of the trajectory. The path should be free of collisions and satisfy a combination of certain optimization criterions, done by the following fitness function:

$$
F F(T)=\operatorname{Ability}(T, O)+\operatorname{Safety}(T, O) / \operatorname{Dist}(T)
$$

where $\operatorname{Dist}(T)$ is the sum of the segments length of the path $T$ and measures the path length. As it is present in the denominator of the second term (1), the higher its value more decreases the fitness value (2). Safety function produces a value that reflects the higher or lower distance between the path and the obstacles $O$ and is given by:

$$
\operatorname{Safety}(T, O)=S_{\max }\left(1-\frac{1}{n} \sum_{s \in T} e^{-D_{s} / \sigma}\right)
$$

where $D_{s}$ is the minimal distance of separation between the segment $s \subset T$ and the set of $O$ segments. It is a near null value if all trajectory segments are very close to the obstacles and it is $S_{\max }$ if all segments of $T$ are fairly distant from segments $O$ (i.e., for $D_{s} \gg \sigma$ ).

The Ability function is a no-collision measure:

$$
\operatorname{Ability}(T, O)=A_{\max }\left(1-\frac{x}{n o}\right)
$$

where $x$ is the number of cross overlapping between the $T$ and $O$ segment's. It is a null value if all segments of the path collide with obstacles $(x=n o)$ and it has a maximum value Amax for a no-collision path $(x=0)$.

According to this definition, the present path planning problem is categorized as an optimization problem, which is solved by GA and SAGA algorithms. They will be used to find the best waypoints at right sequence to define the optimal path, being this process realized into a continuous space. Moreover, the choice of node $i$ is not separable from the choice made for the previous one, i-1. Both algorithms used the above fitness function. The best solution is chosen from all solutions that have a maximum value of fitness. The following parameters values were used: $A_{\max }=10$; $S_{\max }=20, \sigma=5 ;$ no=8 and $\mathrm{n}=10$.

\section{RESUlts}

Conventional GA and SAGA algorithms are used to solve the same complex path-planning problem. Both test results are presented and discussed in this section.

The workspace has a square frontier border with 100 units of length side and it has eight objects inside. All geometrical components are described in two dimensions (2D). The path is limited to 10 waypoints (nodes) inside the workspace. The population size of GA and SAGA is fifty individuals. Each experiment is executed until the 50th generation and repeated in 20 trials. The statistical results here presented are the mean value of these individual tests.

Fig. 1 shows the GA results of the 20 trials, where the red line represents the best of them, although this solution is not the optimal global path. From analysis of the workspace drawn in the figure, it is visible that there are various viable paths connecting the start with the end-point, all of them with a performance near optimal. In this context, GA reveals difficulty to find stable and soft paths. Moreover, it reveals a low rate of generational improvement, as the Fig. 2 shows, where are plotted the mean values of all trials for each generational phase. There are curves for the values of the best, the worst and the mean performance values of population in each generation. These results show that at the end of the 50th generation the mean performance of individuals of GA populations is still far to the maximum, which is also far from the optimal value (the optimal path). The best solution has a fitness value of 11.06 .

In Fig. 3 are plotted the path solutions obtained with the SAGA algorithm for 20 trials. The red line is the optimal 
path, with fitness value 11.11, which circumvents the obstacles safely with a minimum length path. The rate of generational convergence is greater than GA case, as it is shown in Fig. 4. Moreover, all three curves (Min, Max and Mean) converge to the optimal value around 11.

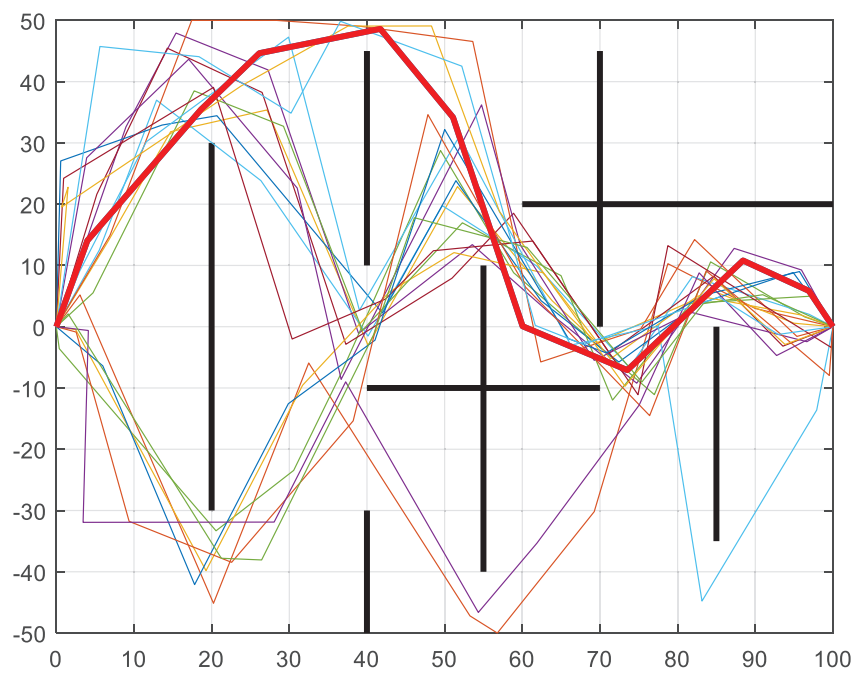

Fig. 1. Best GA path solutions of 20 trials experiments. Red lines the best solution of them.

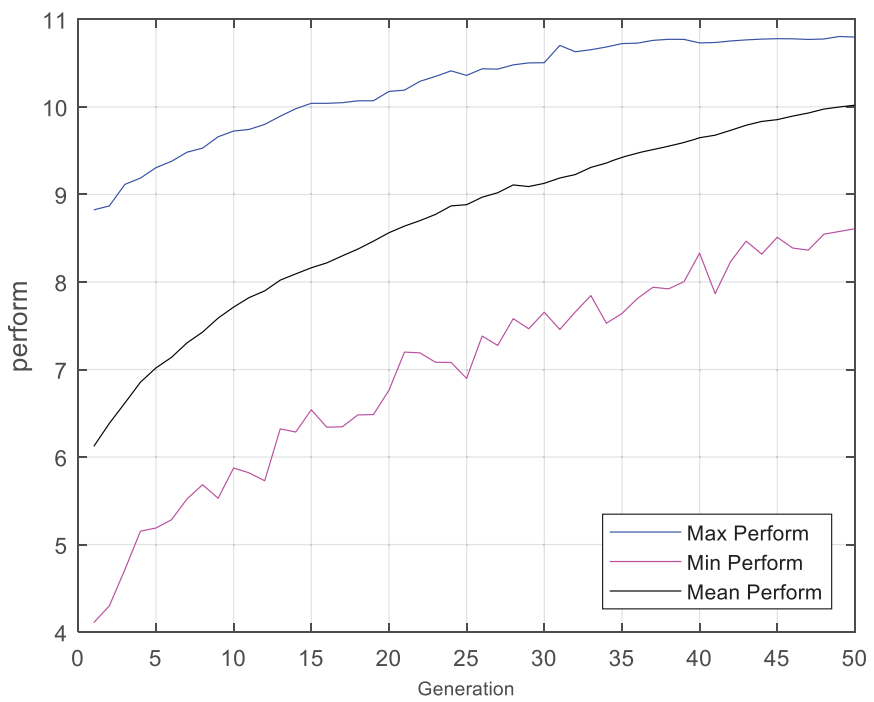

Fig. 2. Mean values of Max, Min and Mean Population performs over generation (for 20 trials experiments) with GA.

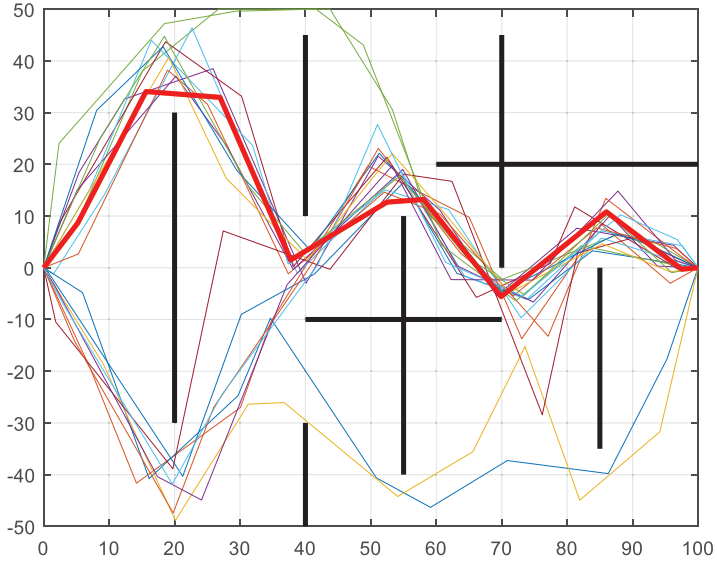

Fig. 3. Best SAGA path solutions of 20 trials experiments. Red lines the best solution of them.

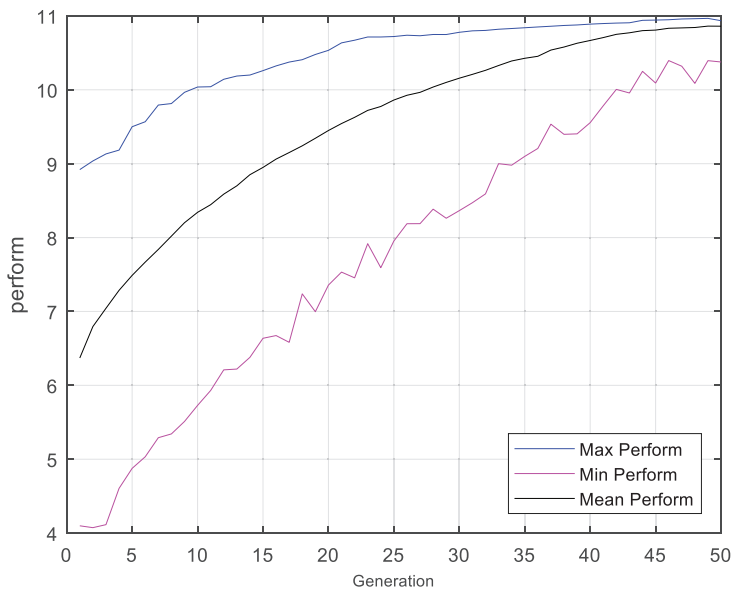

Fig. 4. Mean values of Max, Min and Mean Population performs over generation (for 20 trials experiments) with SAGA.

\section{CONCLUSIONS}

In this paper, a new Genetic Algorithm is proposed for solving the path-planning problem. It is a generalization of GA algorithms, by taking genes as the basic elements. The individuals are seen as the carriers of them and their identity results from the aggregation effects of their enabled genes. The fitness of the individual reveals only the combined behaviour of their active genes in tests during their life, maybe in casual circumstances. Genes are also measured in SAGA algorithms evaluating them in all population. With this information a selfish process occurs imbued in a known mechanism of natural evolution.

SAGA algorithm was shown to be better than GA and it presented best generational convergence and traps avoidance in the search space, namely in a complex path-planning problem.

\section{REFERENCES}

[1] Dan Simon, Evolutionary Optimization Algorithms, John Wiley \& Sons, Inc, 2013

[2] John Henry Holland, Adaptation in Natural and Artificial Systems: An Introductory Analysis with Applications to Biology, Control, and Artificial Intelligence, MIT Press, Bradford Books Editions, (Reprint, ISBN 978-0262581110, 1992), 1975.

[3] B.Y. Qu, Y.S. Zhu, Y.C. Jiao, M.Y. Wu, P.N. Suganthan, J.J. Liang, A survey on multi-objective evolutionary algorithms for the solution 
of the environmental/economic dispatch problems, Swarm and Evolutionary Computation, Vol 38, pp. 1-11, 2018

[4] Ivan Zelinka, A survey on evolutionary algorithms dynamics and its complexity - Mutual relations, past, present and future, Swarm and Evolutionary Computation, Vol 25, pp. 2-14, 2015

[5] T. Ryan Gregory, Understanding Natural Selection: Essential Concepts and Common Misconceptions, Evolution: Education and Outreach, Vol. 2, Issue 2, pp 156-175, 2009

[6] Peter Godfrey-Smith, Conditions for Evolution by Natural Selection, Journal of Philosophy 104 (2007): 489-516

[7] David Zeigler, Chapter 2 - Natural Selection, Editor(s): David Zeigler, Evolution, Academic Press, pp. 9-22, 2014.

[8] Kevin Laland, B. Matthews and M. W. Feldman, An introduction to niche construction theory, Evolutionary Ecology, Vol. 30, Issue 2, pp 191-202, 2016.

[9] V. V. Efremov, Equilibrium between Genetic Drift and Migration at Various Mutation Rates: Simulation Analysis, Russian Journal of Genetics, Vol. 41, Issue 9, pp 1055-1058, Sept. 2005.

[10] Burt, A., and Trivers, R., Genes in conflict: the biology of selfish genetic elements, Cambridge, MA: Belknap Press, 2006.

[11] John C. Avise, Chapter 48 - 1976 Selfish Genes, Editor(s): John C. Avise, Conceptual Breakthroughs in evolutionary Genetics, Academic Press, pp. 101-102, (ISBN 9780124201668), 2014.
[12] Okasha, Samir, "Population Genetics", The Stanford Encyclopedia of Philosophy (Fall 2015 Edition), Edward N. Zalta (ed.)

[13] Richard M. Sibly, Robert N. Curnow, Evolution of discrimination in populations at equilibrium between selfishness and altruism, Journal of Theoretical Biology, Volume 313, pp. 162-171, 2012

[14] Harold Demsetz, Seemingly altruistic behavior: selfish genes or cooperative organisms?, Journal of Bioeconomics, Vol. 11, Issue 3, pp 211-221, Dec. 2009.

[15] Kurt Kleiner, The selfish gene that learned to cooperate, New Scientist, Vol. 191, pp. 13, Issue 2564, 2006.

[16] Paulo Salgado, G. Igrejas and P. Afonso, Hybrid PSO-cubic spline for autonomous robots optimal trajectory planning, INES 2017 o 21st International Conference on Intelligent Engineering Systems, pp. 131136, Oct. 20-23, 2017, Larnaca, Cyprus.

[17] Oliver Kramer, Genetic Algorithm Essentials, Studies in Computational Intelligence, Springer, 2017. 